\title{
On the Constancy of Bribe-Proof Solutions*
}

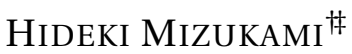 \\ Graduate School of Economics, Osaka University \\ mizukami@iser.osaka-u.ac.jp
}

September 16, 2001

\footnotetext{
*This paper received the Osaka University Institute of Social and Economic Research Moriguchi Prize in January 2001. I am grateful to Prof. Ryoichi Nagahisa, Prof. Tatsuyoshi Saijo, Prof. Ken-ichi Shimomura, Prof. Ken Urai, and especially two anonymous referees for their useful and helpful comments and suggestions. All remaining errors are mine.

${ }^{\dagger}$ Research Fellow of the Japan Society for the Promotion of Science.

${ }^{\ddagger}$ Institute of Social and Economic Research, Osaka University, 6-1 Mihogaoka, Ibaraki, Osaka 567-0047, JAPAN.
} 


\begin{abstract}
We consider the problem of choosing one point in a set of alternatives when monetary transfers are possible. In this context, Schummer (2000) shows that a social choice function must be a constant function if manipulation through bribes is ruled out. But he requires two kinds of domainrichness conditions. One is either smooth connectedness or the finiteness of the set of alternatives and the other is monotonical closedness. However, dispensing with the former condition, we alternatively prove the same result under a weaker condition than monotonical closedness.
\end{abstract}

Keywords: Social Choice, Strategy-Proofness, Bribe-Proofness, Transferable Utility, Constancy.

JEL Classification Numbers: C70, D71, H40. 


\section{Introduction}

We consider the problem of selecting one point from an interval or a multidimensional set, based on valuations reported by participants, when monetary transfers between two agents are possible. In this environment, Schummer (2000) introduced the concept of bribe-proofness which rules out manipulation through monetary transfers and he proved in Theorem 4 that a social choice function satisfies bribe-proofness if and only if it is a constant function.

In this paper, we generalize Schummer's result. Under a domain-richness condition, we first prove that any bribe-proof rule must be an exclusive rule, where at least one agent always has no influence upon social decisions. Strengthening the condition, we second prove that any bribe-proof rule must be a constant rule, where every agent always does not have any influence upon social decisions. The second theorem implies Schummer's Theorem 4 which he proves under two kinds of domain-richness conditions: (a) either smooth connectedness $^{1}$ or the finiteness of the set of alternatives and (b) monotonical closedness. This is because we alternatively prove the theorem without the former condition and under a weaker condition than monotonical closedness. Our domainrichness condition is defined from the viewpoint of the interconnection among valuation functions rather than the sort of valuation functions admissible for each agent.

In this social choice environment there have been a considerable number of contributions since Moulin's seminal work (Moulin (1980)), where he first characterized the class of peak-only strategy-proof ${ }^{2}$ rules. Recently Ching (1997) offered an alternative characterization of the class. It is now believed that, without monetary transfers, the median voter rule ${ }^{3}$ is preferable because it satisfies strategy-proofness. However, it is also known that the median voter rule is manipulable if monetary transfers are possible. In the case where monetary transfers are possible, even if a rule satisfies strategy-proofness, each agent could be better off by another agent's misrepresentations, so that both agents could benefit by means of monetary transfers if they cooperate with each other.

This paper is organized as follows. In Section 2, we introduce notation and definitions. In Section 3, we provide our results. In Section 4, we state some conclusions.

\footnotetext{
${ }^{1}$ Holmström (1979) provided smoothly connectedness to generalize the characterization of Clark-Groves mechanisms to such domains.

${ }^{2}$ Strategy-proofness is the requirement that each agent cannot be better off by his/her own misrepresentations regardless of the other agents' representations.

${ }^{3}$ The median voter rule is the social choice function that selects the median of the peaks of represented valuations.
} 


\section{Notation and Definitions}

Let $N:=\{1,2, \ldots, n\}$, where $\# N \geq 2$, be the set of agents. Let $X \subset \mathbb{R}^{\ell}$ be the set of alternatives. For each agent $i \in N$, let $m_{i} \in \mathbb{R}$ be agent $i$ 's initial endowment of a numeraire, money, which is used for utility transfers. For each agent $i \in N$, let $t_{i j} \in \mathbb{R}$ be a transfer of the numeraire from agent $i$ to $j$. We assume that each agent has enough money to transfer. ${ }^{4}$

For each agent $i \in N$, let $\Theta_{i} \subset \mathbb{R}$ be a set of agent $i$ 's types, which each prescribe his/her preference over $X$. Each agent $i \in N$ has a continuous valuation function $v_{i}: X \times \Theta_{i} \rightarrow \mathbb{R}$, which associates a real number with each alternative $x \in X$ and each type $\theta_{i} \in \Theta_{i}$. Each agent $i \in N$ is equipped with a quasilinear utility function over $X \times \mathbb{R}$, and utility is fully transferable across agents through money. A domain is a set $\Theta:=\Theta_{1} \times \Theta_{2} \times \cdots \times \Theta_{n}$. A type profile is a list $\theta=\left(\theta_{1}, \theta_{2}, \ldots, \theta_{n}\right) \in \Theta$.

A social choice function is a single-valued function $f: \Theta \rightarrow X$, which associates a non-empty alternative $x \in X$ with each type profile $\theta \in \Theta$.

Now we introduce some properties of social choice functions. The first two properties refer to the influence of agents upon social decisions. To formulate these, we provide additional notation. Given $i \in N$ and $\theta_{-i} \in \Theta_{-i}$, the option set is the set $O_{i}\left(\theta_{-i}\right):=\left\{x \in X \mid x=f\left(\theta_{i}, \theta_{-i}\right)\right.$ for some $\left.\theta_{i} \in \Theta_{i}\right\}$. This is the set of alternatives that agent $i$ can obtain by varying his type when the other agents represent $\theta_{-i}$.

An exclusive rule is a social choice function that always neglects any type represented by at least one agent. A constant rule, which is a special case of the exclusive rule, is a social choice function that does not reflect any type reported by any agent.

Definition 1 (An Exclusive Rule). A social choice function $f$ is exclusive if there exists $i \in N$ such that for all $\theta_{-i} \in \Theta_{-i}, O_{i}\left(\theta_{-i}\right)$ is a singleton.

Definition 2 (A Constant Rule). A social choice function $f$ is constant if, for all $i \in N$ and all $\theta_{-i} \in \Theta_{-i}, O_{i}\left(\theta_{-i}\right)$ is a singleton.

Remark 1. We usually define constant rules as follows: a social choice function $f$ is constant if, for all $\theta, \theta^{\prime} \in \Theta$, we have $f(\theta)=f\left(\theta^{\prime}\right)$. Our definition is equivalent to this.

Next we introduce an incentive compatibility condition, which requires each agent to have no incentive to misrepresent his/her types to benefit whether the other agents misrepresent or not.

Definition 3 (Strategy-Proofness). A social choice function $f$ satisfies strategyproofness if, for all $\theta \in \Theta$ and all $i \in N$, there exists no $\theta_{i}^{\prime} \in \Theta_{i}$ such that

$$
v_{i}\left(f\left(\theta_{i}, \theta_{-i}\right), \theta_{i}\right)<v_{i}\left(f\left(\theta_{i}^{\prime}, \theta_{-i}\right), \theta_{i}\right) .
$$

\footnotetext{
${ }^{4}$ This is because we eliminate the case where an agent cannot bribe another for lack of money.
} 
Finally we provide another incentive compatibility condition, which requires each agent to have no incentive to bribe another agent to misrepresent to jointly benefit, irrespective of what the other agents represent.

Definition 4 (Bribe-Proofness). A social choice function $f$ satisfies bribe-proofness if, for all $\theta \in \Theta$ and all $i, j \in N$, there exists no $t_{j i} \in \mathbb{R}$ and $\theta_{i}^{\prime} \in \Theta_{i}$ such that

$$
\left\{\begin{array}{l}
v_{i}\left(f\left(\theta_{i}, \theta_{j}, \theta_{-i, j}\right), \theta_{i}\right)<v_{i}\left(f\left(\theta_{i}^{\prime}, \theta_{j}, \theta_{-i, j}\right), \theta_{i}\right)+t_{j i} \\
v_{j}\left(f\left(\theta_{i}, \theta_{j}, \theta_{-i, j}\right), \theta_{j}\right)<v_{j}\left(f\left(\theta_{i}^{\prime}, \theta_{j}, \theta_{-i, j}\right), \theta_{j}\right)-t_{j i},
\end{array}\right.
$$

that is, for all $\theta \in \Theta$ and all $i, j \in N$, there exists no $\theta_{i}^{\prime} \in \Theta_{i}$ such that

$$
\begin{array}{r}
v_{i}\left(f\left(\theta_{i}, \theta_{j}, \theta_{-i, j}\right), \theta_{i}\right)+v_{j}\left(f\left(\theta_{i}, \theta_{j}, \theta_{-i, j}\right), \theta_{j}\right) \\
<v_{i}\left(f\left(\theta_{i}^{\prime}, \theta_{j}, \theta_{-i, j}\right), \theta_{i}\right)+v_{j}\left(f\left(\theta_{i}^{\prime}, \theta_{j}, \theta_{-i, j}\right), \theta_{j}\right) .
\end{array}
$$

The concept of bribe-proofness was first defined by Schummer (see Schummer (2000) for a more detailed discussion). Note that bribe-proofness implies strategy-proofness by our choosing $i=j$.

\section{Results}

As Schummer (2000) proves, a social choice function $f$ satisfies bribe-proofness if and only if it is constant. But he requires two assumptions about the domain. One is either smooth connectedness ${ }^{5}$ or the finiteness of the set of alternatives and the other is monotonical closedness. ${ }^{67}$ However, dispensing with the former condition, we alternatively prove it under a weaker condition than monotonical closedness. While Schummer's conditions seem to be defined from the viewpoint of the sort of types admissible for each agent, our condition is defined from

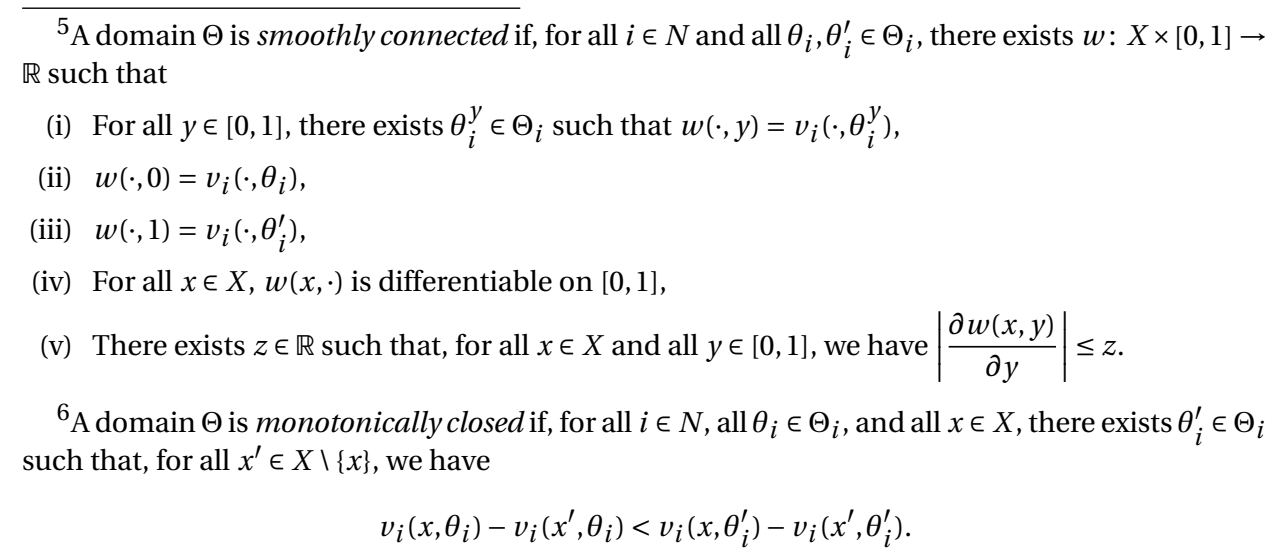

\footnotetext{
${ }^{7}$ These conditions imply an infinite number of types in each type set. However, under the single-peakedness assumption, a finite and small number of types is enough for us to show the constancy of bribe-proof rules (see Example 1 in Mizukami (2000)).
} 
that of the interconnection among types rather than the sort of them. To formulate the condition, we provide additional notation. Given $\theta_{i} \in \Theta_{i}$, the maximal set of $v_{i}\left(\cdot, \theta_{i}\right)$ in $X$ is $\mathscr{P}\left(v_{i}\left(\cdot, \theta_{i}\right)\right):=\arg \max _{x \in X} v_{i}\left(x, \theta_{i}\right)$. When the set is a singleton, we denote its unique element by $p\left(v_{i}\left(\cdot, \theta_{i}\right)\right)$ and call it the peak of $v_{i}\left(\cdot, \theta_{i}\right)$.

The following is our domain-richness condition, which requires that there exists a pair $(i, j)$ satisfying the following: for any distinct alternatives $x, y \in X$, (i) agent $i$ has a relatively steeper valuation function whose peak is $x$ and for which, for any other alternative $x^{\prime} \in X$, the difference of values between $x$ and $x^{\prime}$ is strictly greater than that of values between them for a valuation function of agent $j$ whose peak is $x^{\prime}$, and (ii) agent $j$ also has a relatively steeper valuation function whose peak is $y$ and for which, for any other alternative $y^{\prime} \in X$, the difference of values between $y$ and $y^{\prime}$ is strictly greater than that of values between them for a valuation function of agent $i$ whose peak is $y^{\prime}$.

Definition 5 (Mutual Dominance). A domain satisfies mutual dominance if there exists a pair $(i, j)$ with $i \neq j$ such that, for any distinct $x, y \in X$, there exist $\theta_{i}$ with $p\left(v_{i}\left(\cdot, \theta_{i}\right)\right)=x$ and $\theta_{j}$ with $p\left(v_{j}\left(\cdot, \theta_{j}\right)\right)=y$ satisfying the following:

(i) For $\theta_{i}$ and any $x^{\prime} \in X \backslash\{x\}$, there exists $\theta_{j}^{\prime}$ with $p\left(v_{j}\left(\cdot, \theta_{j}^{\prime}\right)\right)=x^{\prime}$ such that $v_{j}\left(x^{\prime}, \theta_{j}^{\prime}\right)-v_{j}\left(x, \theta_{j}^{\prime}\right)<v_{i}\left(x, \theta_{i}\right)-v_{i}\left(x^{\prime}, \theta_{i}\right)$.

(ii) For $\theta_{j}$ and any $y^{\prime} \in X \backslash\{y\}$, there exists $\theta_{i}^{\prime}$ with $p\left(v_{i}\left(\cdot, \theta_{i}^{\prime}\right)\right)=y^{\prime}$ such that $v_{i}\left(y^{\prime}, \theta_{i}^{\prime}\right)-v_{i}\left(y, \theta_{i}^{\prime}\right)<v_{j}\left(y, \theta_{j}\right)-v_{j}\left(y^{\prime}, \theta_{j}\right)$.

Note that mutual dominance is independent of single-peakedness. In fact, there is a domain which satisfies mutual dominance but does not contain single-peaked valuation functions at all.

Now we introduce an impossibility theorem, which states that any bribeproof rule must be exclusive if a domain satisfies mutual dominance. That is, any bribe-proof rule always excludes at least one agent from the social decision mechanism.

Theorem 1. Suppose that a domain $\Theta$ satisfies mutual dominance. If a social choice function $f$ is bribe-proof it is exclusive.

Proof. Suppose that $f$ is not exclusive, then, for all $h \in N$, there exist $\theta_{-h} \in \Theta_{-h}$ and distinct $x_{h}, x_{h}^{\prime} \in X$ such that $x_{h}, x_{h}^{\prime} \in O_{h}\left(\theta_{-h}\right)$. That is, by the definition of the option set, for all $h \in N$, there exist $\theta_{-h} \in \Theta_{-h}$ and distinct $\theta_{h}, \theta_{h}^{\prime} \in \Theta_{h}$ such that $f\left(\theta_{h}, \theta_{-h}\right)=x_{h}$ and $f\left(\theta_{h}^{\prime}, \theta_{-h}\right)=x_{h}^{\prime}$.

Since the domain satisfies mutual dominance, there exists a pair $(i, j)$ with $i \neq j$ such that, for distinct $f(\theta), f\left(\theta_{i}^{\prime}, \theta_{-i}\right) \in X$, there exist $\bar{\theta}_{i}$ with $p\left(v_{i}\left(\cdot, \bar{\theta}_{i}\right)\right)=$ $f(\theta)$ and $\bar{\theta}_{j}$ with $p\left(v_{j}\left(\cdot, \bar{\theta}_{j}\right)\right)=f\left(\theta_{i}^{\prime}, \theta_{-i}\right)$ satisfying the following:

(i) For $\bar{\theta}_{i}$ and any $\hat{x} \in X \backslash\{f(\theta)\}$, there exists $\hat{\theta}_{j}$ with $p\left(v_{j}\left(\cdot, \hat{\theta}_{j}\right)\right)=\hat{x}$ such that $v_{j}\left(\hat{x}, \hat{\theta}_{j}\right)-v_{j}\left(f(\theta), \hat{\theta}_{j}\right)<v_{i}\left(f(\theta), \bar{\theta}_{i}\right)-v_{i}\left(\hat{x}, \bar{\theta}_{i}\right)$. 
(ii) For $\bar{\theta}_{j}$ and any $\hat{y} \in X \backslash\left\{f\left(\theta_{i}^{\prime}, \theta_{-i}\right)\right\}$, there exists $\hat{\theta}_{i}$ with $p\left(\nu_{i}\left(\cdot, \hat{\theta}_{i}\right)\right)=\hat{y}$ such that $v_{i}\left(\hat{y}, \hat{\theta}_{i}\right)-v_{i}\left(f\left(\theta_{i}^{\prime}, \theta_{-i}\right), \hat{\theta}_{i}\right)<v_{j}\left(f\left(\theta_{i}^{\prime}, \theta_{-i}\right), \bar{\theta}_{j}\right)-v_{j}\left(\hat{y}, \bar{\theta}_{j}\right)$.

We do not change types other than types of agents $i$ and $j$, so we describe type profiles $\left(\cdot, \cdot, \theta_{-i, j}\right)$ as $(\cdot, \cdot)$ hereafter.

By strategy-proofness, agent $i$ 's changes from $(\theta)$ to $\left(\bar{\theta}_{i}, \theta_{j}\right)$ and vice versa imply $f\left(\bar{\theta}_{i}, \theta_{j}\right)=f(\theta)$; otherwise, because $p\left(\nu_{i}\left(\cdot, \bar{\theta}_{i}\right)\right)=f(\theta)$ we have $v_{i}\left(f\left(\bar{\theta}_{i}, \theta_{j}\right), \bar{\theta}_{i}\right)<$ $v_{i}\left(f(\theta), \bar{\theta}_{i}\right)$, which is contradicting strategy-proofness. Similarly, by strategyproofness, agent $j$ 's changes from $\left(\theta_{i}^{\prime}, \theta_{j}\right)$ to $\left(\theta_{i}^{\prime}, \bar{\theta}_{j}\right)$ and vice versa imply $f\left(\theta_{i}^{\prime}, \bar{\theta}_{j}\right)=$ $f\left(\theta_{i}^{\prime}, \theta_{j}\right)$; otherwise, because $p\left(v_{j}\left(\cdot, \bar{\theta}_{j}\right)\right)=f\left(\theta_{i}^{\prime}, \theta_{j}\right)$ we have $v_{j}\left(f\left(\theta_{i}^{\prime}, \bar{\theta}_{j}\right), \bar{\theta}_{j}\right)<$ $v_{j}\left(f\left(\theta_{i}^{\prime}, \theta_{j}\right), \bar{\theta}_{j}\right)$, which is contradicting strategy-proofness.

Step 1: $f\left(\bar{\theta}_{i}, \theta_{j}\right)=f\left(\bar{\theta}_{i}, \bar{\theta}_{j}\right)$.

Suppose not, then, by the definition of mutual dominance, for $\bar{\theta}_{i}$ with $p\left(v_{i}\left(\cdot, \bar{\theta}_{i}\right)\right)=$ $f(\theta)=f\left(\bar{\theta}_{i}, \theta_{j}\right)$ and $f\left(\bar{\theta}_{i}, \bar{\theta}_{j}\right) \in X \backslash\left\{f\left(\bar{\theta}_{i}, \theta_{j}\right)\right\}$, there exists $\tilde{\theta}_{j}$ with $p\left(v_{j}\left(\cdot, \tilde{\theta}_{j}\right)\right)=$ $f\left(\bar{\theta}_{i}, \bar{\theta}_{j}\right)$ such that

$$
v_{j}\left(f\left(\bar{\theta}_{i}, \bar{\theta}_{j}\right), \tilde{\theta}_{j}\right)-v_{j}\left(f\left(\bar{\theta}_{i}, \theta_{j}\right), \tilde{\theta}_{j}\right)<v_{i}\left(f\left(\bar{\theta}_{i}, \theta_{j}\right), \bar{\theta}_{i}\right)-v_{i}\left(f\left(\bar{\theta}_{i}, \bar{\theta}_{j}\right), \bar{\theta}_{i}\right) .
$$

By strategy-proofness, agent $j$ 's changes from $\left(\bar{\theta}_{i}, \bar{\theta}_{j}\right)$ to $\left(\bar{\theta}_{i}, \tilde{\theta}_{j}\right)$ and vice versa imply $f\left(\bar{\theta}_{i}, \tilde{\theta}_{j}\right)=f\left(\bar{\theta}_{i}, \bar{\theta}_{j}\right)$; otherwise, because $p\left(v_{j}\left(\cdot, \tilde{\theta}_{j}\right)\right)=f\left(\bar{\theta}_{i}, \bar{\theta}_{j}\right)$ we have $v_{j}\left(f\left(\bar{\theta}_{i}, \tilde{\theta}_{j}\right), \tilde{\theta}_{j}\right)<v_{j}\left(f\left(\bar{\theta}_{i}, \bar{\theta}_{j}\right), \tilde{\theta}_{j}\right)$, which is contradicting strategy-proofness. Therefore, by (1),

$$
\begin{gathered}
v_{j}\left(f\left(\bar{\theta}_{i}, \tilde{\theta}_{j}\right), \tilde{\theta}_{j}\right)-v_{j}\left(f\left(\bar{\theta}_{i}, \theta_{j}\right), \tilde{\theta}_{j}\right)<v_{i}\left(f\left(\bar{\theta}_{i}, \theta_{j}\right), \bar{\theta}_{i}\right)-v_{i}\left(f\left(\bar{\theta}_{i}, \tilde{\theta}_{j}\right), \bar{\theta}_{i}\right) \\
v_{i}\left(f\left(\bar{\theta}_{i}, \tilde{\theta}_{j}\right), \bar{\theta}_{i}\right)+v_{j}\left(f\left(\bar{\theta}_{i}, \tilde{\theta}_{j}\right), \tilde{\theta}_{j}\right)<v_{i}\left(f\left(\bar{\theta}_{i}, \theta_{j}\right), \bar{\theta}_{i}\right)+v_{j}\left(f\left(\bar{\theta}_{i}, \theta_{j}\right), \tilde{\theta}_{j}\right),
\end{gathered}
$$

which is contradicting bribe-proofness.

Step 2: $f\left(\theta_{i}^{\prime}, \bar{\theta}_{j}\right)=f\left(\bar{\theta}_{i}, \bar{\theta}_{j}\right)$.

By an argument similar to Step 1, we can prove Step 2.

Thus, we have $f(\theta)=f\left(\bar{\theta}_{i}, \theta_{j}\right)=f\left(\bar{\theta}_{i}, \bar{\theta}_{j}\right)=f\left(\theta_{i}^{\prime}, \bar{\theta}_{j}\right)=f\left(\theta_{i}^{\prime}, \theta_{j}\right)$ : a contradiction because $f(\theta) \neq f\left(\theta_{i}^{\prime}, \theta_{j}\right)$.

Finally, strengthening Definition 5, we introduce another sort of impossibility theorem, which corresponds to Theorem 4 in Schummer (2000). The distinction between Definitions 5 and 6 is the number of pairs required to satisfy Conditions (i) and (ii).

Definition 6 (Strong Mutual Dominance). ${ }^{8}$ A domain satisfies strong mutual dominance if for all $i \in N$ there exists a partner $j \in N \backslash\{i\}$ such that, for any distinct $x, y \in X$, there exist $\theta_{i}$ with $p\left(v_{i}\left(\cdot, \theta_{i}\right)\right)=x$ and $\theta_{j}$ with $p\left(v_{j}\left(\cdot, \theta_{j}\right)\right)=y$ satisfying Conditions (i) and (ii) in Definition 5.

\footnotetext{
${ }^{8}$ This condition is similar to dual dominance provided by Saijo (1987), where he proved that Maskin monotonic social choice functions must be constant if the domain satisfies dual dominance.
} 
Note that this condition is implied by monotonical closedness, which requires that, for any valuation function and any alternative $x \in X$, there exists another valuation function for which the value of $x$, relative to any other alternative, is strictly greater than that of $x$ for the original function. Accordingly, by definition, monotonical closedness implies that the differences of valuations between any two alternatives in $X$ are not each bounded. Then, for any alternative $x \in X$, each agent has a relatively steeper valuation function, the maximal set of which is $\{x\}$ and for which, for any other alternative $x^{\prime} \in X$, the difference of values between $x$ and $x^{\prime}$ is strictly greater than that of values between them for a valuation function of each of the other agents, the maximal set of which is $\left\{x^{\prime}\right\}$. Therefore strong mutual dominance is implied by monotonical closedness.

In Theorem 1, the condition of mutual dominance requires at least one agent to partner another, so that the bribe-proof rule reflects no type represented by the pair. Correspondingly, in Theorem 2, the condition of strong mutual dominance requires each agent to partner another, so that the bribe-proof rule neglects any type reported by the pairs, that is, by every agent.

Theorem 2. Suppose that a domain $\Theta$ satisfies strong mutual dominance. A social choice function $f$ is bribe-proof if and only if it is constant.

By an argument similar to Theorem 1, we can prove Theorem 2. Note that our dominance conditions are both compatible with the finiteness of $X$. Therefore our theorems hold in the case where $X$ is finite.

\section{Conclusion}

In this paper, we have provided a generalization of Schummer's Theorem 4 which states that a social choice function satisfies bribe-proofness if and only if it is a constant function. Our main result, Theorem 2, differs from his Theorem 4 on two points. First, whereas he requires two kinds of domain-richness conditions, we alternatively prove it under the strong mutual dominance condition which is implied by his one condition and without the other condition. Furthermore, he assumes that the set of alternatives is compact, whereas we do not. Second, our condition holds whether the set of alternatives is infinite or not, while his conditions do not. Exploring whether we can design a non-trivial bribe-proof rule in other models would be an important topic for further investigation. 


\section{References}

ChIng, S. (1997): "Strategy-Proofness and "Median Voters", International Journal of Game Theory, 26, 473-490.

Holmström, B. (1979): “Groves' Scheme on Restricted Domains," Econometrica, 47, 1137-1144.

Mizukami, H. (2000): "On the Constancy of Bribe-Proof Solutions," mimeo., Osaka University.

Moulin, H. (1980): "On Strategy-Proofness and Single-Peakedness," Public Choice, 35, 437-455.

SAIJO, T. (1987): "On Constant Maskin Monotonic Social Choice Functions," Journal of Economic Theory, 42, 382-386.

SChummer, J. (2000): “Manipulation through Bribes," Journal of Economic Theory, 91, 180-198. 